\title{
Grains and Strains from Cepstral Analysis of 4D-STEM Nano-Diffraction Datasets
}

Elliot Padgett ${ }^{1}$, Paul Cueva ${ }^{1}$, Megan E. Holtz ${ }^{1,2}$, Eric Langenberg ${ }^{2}$, Dong Ren ${ }^{3}$, Héctor D. Abruña ${ }^{3}$, Darrell Schlom ${ }^{2}$, and David A. Muller ${ }^{1,4}$

1. School of Applied and Engineering Physics, Cornell University, Ithaca, NY.

2. Department of Materials Science and Engineering, Cornell University, Ithaca, NY.

3. Department of Chemistry and Chemical Biology, Cornell University, Ithaca, NY.

4. Kavli Institute at Cornell for Nanoscale Science, Cornell University, Ithaca, NY.

The availability of fast, high dynamic range pixel array detectors [1] makes scanning convergent beam electron diffraction (CBED) a powerful tool for rapid, spatially resolved characterization of lattice structure over a wide range of length scales. However, to extract useful structural information from the large 4-dimensional datasets, especially for complex, 3D specimens, we must confront challenges with CBED intensity variations due to crystal mistilts and multiple scattering as well as challenges with precisely localizing diffracted disk centers.

Applying physically motivated transformations can improve the interpretability of scanning diffraction data and reduce its sensitivity to unwanted artifacts. Here we use cepstrum analysis [2] to provide a more direct measurement of the lattice structure. By computing the power cepstrum of the exit wave we separate the effects of mistilt from the underlying lattice information. The exit wave power cepstrum (EWPC) provides an effective basis for component analysis of specimens with large mosaic spreads and mistilts caused by crystal defects, such as the $\mathrm{LiNi}_{x} \mathrm{Mn}_{y} \mathrm{Co}_{z} \mathrm{O}_{2}$ (NMC) battery cathode particle shown in Figure 1. A false color map of principal component analysis (PCA) concentrations performed in the EWPC space effectively reveals the grain structure of the specimen without significant contrast from defect-induced mistilts that would plague a traditional PCA analysis of the same dataset.

The EWPC space is also effective for quantitative measurement of lattice distortions. In the EWPC space, the signal can be viewed as a weighted Patterson function, with localized peaks corresponding to lattice spacings. By fitting these peaks, the lattice parameters, rotation, and strain can be extracted in maps at sub-nanometer spatial resolution with $\sim 1 \mathrm{~ms} /$ pixel dwell time. This is demonstrated for an epitaxial film of $\mathrm{PbTiO}_{3}$ (PTO) strained on a $\mathrm{DyScO}_{3}$ (DSO) substrate (Figure 2). The PTO accommodates this strain with alternating domains, where the long axis of the tetragonal unit cell orients either in the growth direction or in the plane of the film. By fitting peaks in the EWPC corresponding to $d_{001}$ and $d_{100}$, we extract the lattice constant in the growth direction $\left(a_{z}\right)$ and in the film plane $\left(a_{x}\right)$, as well as the lattice rotation and shear strain. These maps show the alternating domain structure as well as subtle strains at the domain walls.

Cepstrum analysis and other physically motivated transformations for scanning CBED provide significant advantages in selecting signals of physical interest, without interference from unwanted features, and by reducing non-linearities, providing datasets well suited for modern linear processing [3]. 
References:

[1] MW Tate et al, Microscopy and Microanalysis 22 (2016), p. 237.

[2] AM Noll, The Journal of the Acoustical Society of America 41 (1967), p. 293.

[3] E.P. acknowledges support from a NSF Graduate Research Fellowship (DGE-1650441) and DOE EERE (DE-EE0007271). M.E.H. supported by DOE-BES (DE-SC0002334). Electron microscopy facility support from NSF MRSEC program (DMR-1719875) and NSF MRI grant (DMR-1429155).
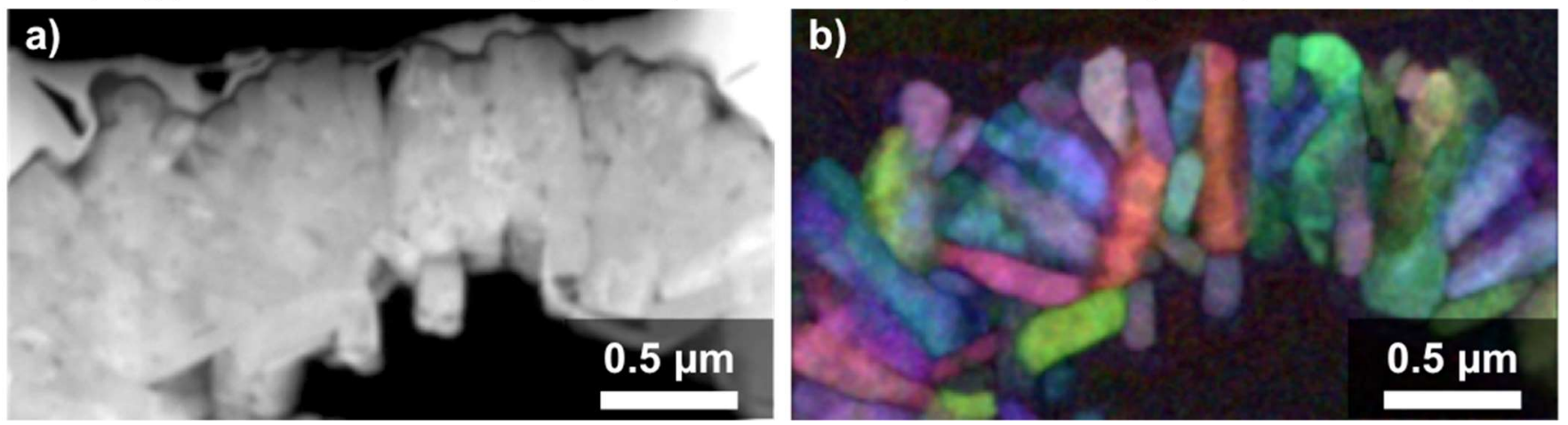

Figure 1. (a) Annular dark field image of a thinned NMC battery cathode particle and (b) a false-color grain map of the specimen produced by colorizing PCA components of the power cepstrum map.

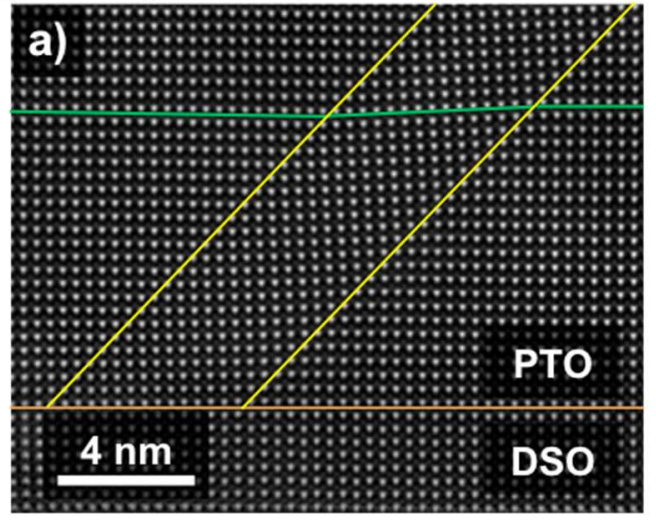

d)

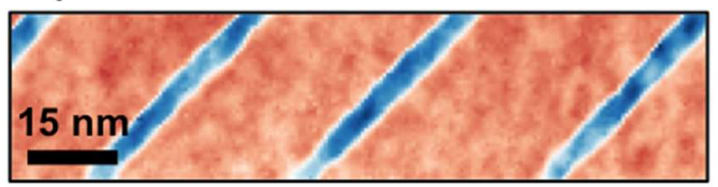

f)

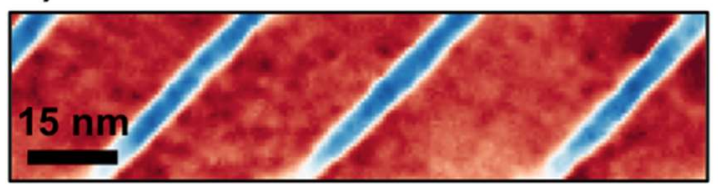

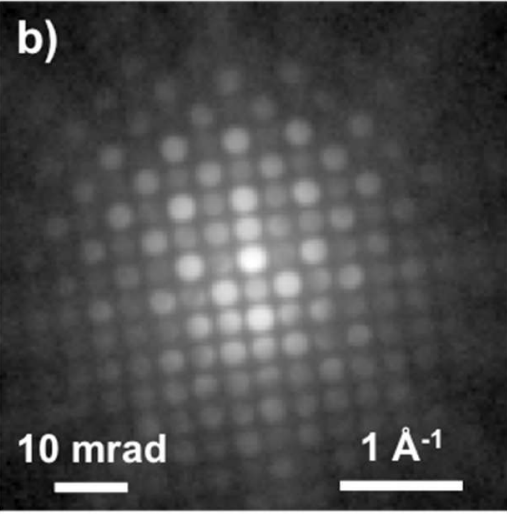

e)

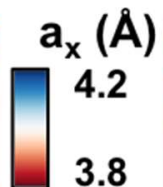

3.8
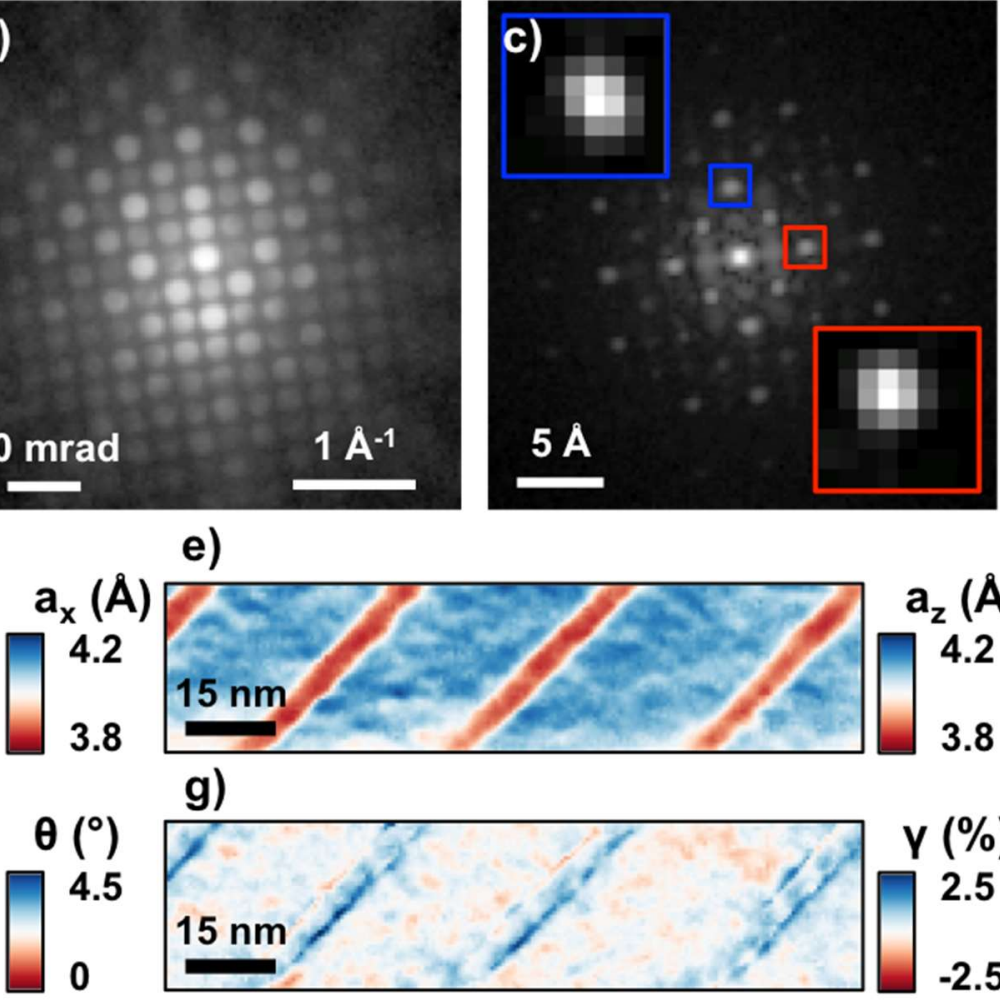

$a_{z}(\AA)$

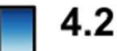

3.8

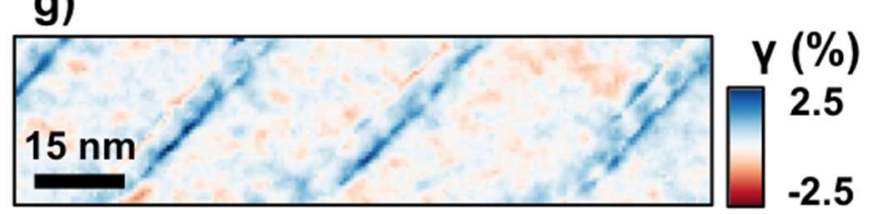

Figure 2. (a) Aberration-corrected HAADF STEM image of the PTO film on the DSO substrate, showing alternating tetragonal lattice domains. (b) Position-averaged CBED pattern from one domain of the PTO film, and (c) the position-averaged power cepstrum (shown in log scale) for the same region, with linear-scale insets showing the 100 and 001 peaks used for fitting. Quantitative lattice structure maps showing the lattice constant in the film plane (d), in the growth direction (e), the lattice rotation (f), and the shear strain (g). 